\title{
Robust Precision Control for a Class of Electro-Hydraulic Actuator System based on Disturbance Observer
}

\author{
Zulfatman Has', Mohd. Fua'ad Rahmat',", Abdul Rashid Husain', and Mohd. Noh Ahmad' \\ 1 Department of Control and Mechatronics Engineering, Faculty of Electrical Engineering, Universiti Teknologi Malaysia Johor Bahru, Skudai, Johor, 81310, Malaysia \\ \# Corresponding Author / E-mail: fuaad@fke.utm.my, TEL: +607-555-7023, FAX: +607-5566272
}

KEYWORDS: Precision tracking control, Sliding mode, Disturbance observer, Electro-hydraulic actuator

\begin{abstract}
This paper presents a new robust control scheme for a class of electro-hydraulic actuator using dynamic sliding mode control associated with nonlinear disturbance observer. Switching-gain of the sliding mode is designed to be adaptable on the estimated disturbance. A switching-gain adaptation mechanism is proposed to obtain as small as possible switching-gain to minimize chattering effect. The scheme is developed to guarantee the tracking precision of the system with robust and smooth control actions in the existence of uncertainties and the changes of external disturbance. Capability of the proposed scheme is enhanced by varying boundary layers algorithm to assist the scheme to return to its ability in a larger change of external disturbance. Capability and effectiveness of the proposed scheme are validated through experiment, where the results indicate that the proposed scheme ensures the tracking precision of the system with robust and smooth control actions in a large change of external load disturbance. Moreover, smooth control actions that are produced by the proposed control scheme offer a significant efficiency of energy in the control of electro-hydraulic actuator systems.
\end{abstract}

\section{NOMENCLATURE}

$A_{1}, A_{2}=$ Cross section area of the two chambers $\left(\mathrm{m}^{2}\right)$

$a_{p}=$ Piston acceleration $\left(\mathrm{m} / \mathrm{s}^{2}\right)$

$C_{d}=$ Discharge coefficient

$C_{v 1}, C_{v 2}=$ Valve orifice coefficients

$d=$ External load disturbance

$D=$ Lumped disturbance

$e=$ Error trajectory

$f=$ Nonlinear dynamics

$F_{a}=$ Hydraulic actuating force $(\mathrm{N})$

$F_{f}=$ Hydraulic friction force $(\mathrm{N})$

$f_{d}=$ Lumped uncertain nonlinearities

$g=$ Control gain

$g_{\text {min }}, g_{\text {max }}=$ Lower and upper bounds of the control gain

$k=$ Spring constant $(\mathrm{N} / \mathrm{m})$

$K=$ Reaching time function

$k_{a}=$ Servo valve gain $(\mathrm{m} / \mathrm{V})$

$k_{v}=$ Viscous friction $(\mathrm{N} \mathrm{s} / \mathrm{m})$

$L=$ Disturbance observer gain

$m=$ Total mass of the piston and load $(\mathrm{kg})$ $p_{1}, p_{2}=$ Pressure in chambers 1 and $2(\mathrm{~Pa})$

$Q=$ Discontinuous switching gain

$S=$ Sliding surface

$u, u_{e q}, u_{n}=$ Total, equivalent and switching control (V)

$V=$ Lyapunov function

$v_{p}=$ Piston velocity $(\mathrm{m} / \mathrm{s})$

$V_{1}, V_{2}=$ Total volume in chambers 1 and $2\left(\mathrm{~m}^{3}\right)$

$x_{d}=$ Desired position $(\mathrm{m})$

$x_{p}=$ Piston position (m)

$\beta_{e}=$ Effective bulk modulus $(\mathrm{Pa})$

$\phi=$ Thickness of boundary layer

$\rho=$ Fluid mass density $\left(\mathrm{kg} / \mathrm{m}^{3}\right)$

\section{Introduction}

Electro-hydraulic actuator (EHA) systems play an important role as precision positioning equipments in industrial robots, aircraft flightcontrol, automotive equipments, and automated manufacturing systems. Tracking precision control has become one of the most 
intensive studies in the control of EHA systems. ${ }^{1-6}$ However, the precision control has been challenging due to the fact that EHA systems exhibit the highly nonlinear properties which include servo valve flow pressure, control fluid volumes, internal leakage, dead zone, stiffness and associated friction. These properties may cause large parameters variations, unmodeled dynamics, or process disturbances which are identified as parametric uncertainties and uncertain nonlinearities. ${ }^{7}$ Apart from these properties EHA systems also have a large extent of model uncertainties due to external disturbance.

Nonlinear robust control techniques have become popular approaches for systems containing uncertainties and disturbances. ${ }^{8}$ In the class of robust control, sliding mode control (SMC) is envisaged to be an effective strategy for a specific class of nonlinear systems. SMC can completely counteract uncertainties and disturbances when the state is constrained to the sliding surface which satisfies the matching condition. ${ }^{9}$ It provides a systematic method to maintain the stability, robust to parameters variations, and satisfactory performance despite of modeling imperfections. ${ }^{10}$ Pertaining to these advantages, various approaches of SMC have been successfully applied to EHA systems for the friction compensation, ${ }^{11}$ flexible load, ${ }^{12}$ and variations in the original control volumes. ${ }^{13}$

In conventional SMC, a discontinuous control is employed based on the bounds of uncertainties and disturbances to guarantee a closed-loop system insensitive to uncertainties and disturbances. However, due to the complexity of the structure of uncertainties and disturbances, these bounds may not be easily obtained. Over-estimation on the bounds may cause unnecessarily large control activity and could possibly damage actuators. Therefore, SMC designs with an adaptation parameter in the control law that is adaptable on the bounds were an effective solution for the requirement to know the bounds explicitly.

Adaptation law technique for upper bounds on the norm of uncertainty using boundary layer was the early proposed technique in the adaptive form of SMC. ${ }^{14}$ In this adaptation law, the switching gain increases whenever the switching function do not convergence to zero. To solve the unbounded growth of the switching gain, a modified adaptation law was developed by Wheeler et al. ${ }^{15} \mathrm{~A}$ unique artificial neural network using 2-sigma network also was applied to estimate upper and lower bounds of uncertainty adaptively. ${ }^{16}$ These bounds provide the switching-gain adjusted to result smoother control action. Similarity of those techniques is limitation of their capability to observe the existence of disturbances. Following this, uncertainty and disturbance estimator (UDE) was developed for the control of linear time invariant that have significant uncertainties in the control input matrix and disturbances. ${ }^{17}$ Another technique was the use of nonlinear disturbance observer (NDO) with an appropriate gain function to approximate the unknown disturbance for a nonlinear system. ${ }^{18}$ However, in the proposed technique NDO was not used to update the switching gain and only be part of equivalent control. It could minimize chattering, but may degrade tracking precision and control stability. Thus, it may not be suitable for a large external disturbance.

For EHA systems that demonstrate strong effects of nonlinear behaviors and a large external disturbance, the use of NDO integrated with SMC using a modified adaptation law might be a realistic choice. The problem addressed in the design is a control law with as small as possible of switching-gain. The gain is required only to be greater than the bounds of estimated disturbance. Therefore, an adaptation law needs to be created to automatically tune the switching-gain. However, this adaptation law might not fully guarantee the observer error converges to zero, when a large change of external disturbance exists. It causes as small as possible chattering cannot be reached. To solve this, a varying boundary layers algorithm can be employed based on the estimated disturbance error.

A novel idea in the above preliminary discussion is selected to be the proposed control strategy in this work. There are three main contributions of this paper. The first one is the integration NDO into SMC with new switching-gain adaptation mechanism to improve robustness and smoothness of the control action with guaranteed tracking performance in the change of external disturbance. The second contribution is the effectiveness of the proposed varying boundary layers algorithm in a larger change of external disturbance. The last contribution of this paper is the use of the proposed scheme for a class EHA system. A smooth control action that is produced by the proposed scheme offers a significant efficiency of energy in the control of EHA systems. Capability and effectiveness of the proposed scheme are verified from experiment results. The rest of the paper is organized as follows. The next section describes the EHA system model form into a class of nonlinear system. In the third section, the proposed control scheme is presented. Then, section four is overview on the simulation and experiment set-up. The scheme needs to finely-tuned by simulation. Then the simulated scheme is verified to the EHA system test-bed. The fifth section is a series of discussion on the experiment results through a comparison to the conventional scheme. The last section would be conclusion of the study.

\section{EHA System Formulation}

The EHA system is double-acting hydraulic cylinder with single ended piston. A single load attached at the end of the piston without spring and damper. ${ }^{19}$ If the state variables of the system are selected as $x=\left[x_{1}, x_{2}, x_{3}\right]^{T}=\left[x_{p}, v_{p}, a_{p}\right]^{T}$, state equations of the system is third order associated to position, velocity and acceleration as follows:

$$
\begin{gathered}
\dot{x}_{1}(t)=x_{2}(t) \\
\dot{x}_{2}(t)=x_{3}(t) \\
\dot{x}_{3}(t)=\dot{a}_{p}=\frac{1}{m}\left[\left(A_{1} \dot{p}_{1}-A_{2} \dot{p}_{2}\right)-\dot{F}_{f}-k \dot{x}_{1}-\dot{f}_{d}\right]
\end{gathered}
$$

Using the EHA system model in Ref. 20-22, the dynamic system equation in Eq. (1) can be rewritten as single input single output nonlinear system as follows

$$
\dot{x}_{3}(t)=f(x)+g u(t)+d(t)
$$

where, $f(x)=\left(\left(-\frac{\beta_{e}}{m}\left(\frac{A_{1}^{2}}{V_{1}}+\frac{A_{2}^{2}}{V_{2}}\right)-\frac{k}{m}\right) x_{2}-\frac{\dot{F}_{f}}{m}\right)$,

$g(x)=\frac{\beta_{e} C_{v} k_{a}}{m}\left(\frac{A_{1}}{V_{1}} \sqrt{\Delta p_{1}}+\frac{A_{2}}{V_{2}} \sqrt{\Delta p_{2}}\right), d(t)=-\frac{\dot{f}_{d}}{m}$

The derivative of friction force, $F_{f}$ in Eq. (1) represents a complex and a natural phenomenon of friction. Friction always appears in 
hydraulic systems especially in cylinder rod and load environment. ${ }^{23}$ In order to accommodate all behaviors in static and dynamic friction together, the friction force is modeled using LuGre friction model. ${ }^{24}$ This complete friction model is represented by four static parameters and two dynamic parameters, stiffness and damping coefficient.

\section{Controller Design}

Consider the nonlinear system equation described in control canonical form Eq. (2), we have

$$
\dot{x}_{3}(t)=\hat{f}(x)+(\Delta f)(x, t)+g u(t)+d(t)
$$

where, the function $\hat{f}(x)$ is nominal part of nonlinear dynamics of the system, $f(x, t)$ is uncertainties, $u(t)$ is control input of the system, and term $d(t)$ is external load disturbance and modeling inaccuracy.

Following Eq. (3), the nonlinear dynamics uncertainty $f(x, t)$ can be treated as part of unknown disturbance and lumped all together with external disturbance $d(t)$ to be

$$
D \geq \Delta f(x, t)+d(t)
$$

where, $D$ represents a time-varying lumped disturbance.

Considering Eq. (3), the nonlinear system equation can be rewritten as

$$
\dot{x}_{3}(t)=\hat{f}(x)+g(x) u(t)+D
$$

In the same manner, the control input gain $g(x)$ is also not known, because some parameters are varying under different operating condition. Thus, uncertainty is accordingly bounded to ensure that control gain does not cause control failure. This is given by following inequality.

$$
0<g_{\text {min }} \leq g \leq g_{\text {max }}
$$

$g_{\min }$ and $g_{\max }$ be known as upper and lower bounds of $g$. In the dynamics, the control input is multiplied by the control gain and the geometric mean of the upper and lower bounds of the gain are taken as an estimation of $g$, such that

$$
\hat{g}=\left(g_{\min } \cdot g_{\max }\right)^{1 / 2}
$$

\subsection{Adaptive sliding mode control}

The goal of this control design is to achieve a continuous sliding control, $u$ such that the output of the system $x_{p}$ tracks the desired input $x_{d}$ as closely as possible.

In designing a desired trajectory, the state error of the system is defined as in ${ }^{16}$

$$
e_{i}=x_{i}-x_{i d} \quad \text { where } i=1 \text { to } 3
$$

The designing of proposed control consists of selecting an equilibrium manifold or sliding surface, $S(x)$ to prescribe the desired dynamic characteristic of the controlled system, and designing a control law to derive the system to the sliding mode $S(x)=0$ and to maintain it on the surface all the time. ${ }^{25}$ In order to achieve the states of the system to track the desired trajectories at the same time, the function of sliding surface is defined in ${ }^{2}$ as follows

$$
S(e, t)=\left(\frac{d}{d t}+\lambda\right)^{2} e_{1}=\lambda^{2} e_{1}+2 \lambda e_{2}+e_{3}
$$

$\lambda$ is a strictly positive constant to be specified according to the desired dynamics of the closed-loop system. The desired dynamic response for the system is given as $S=0$. If $S$ is forced to zero, the desired dynamics is attained and consequently, the tracking error will converge to zero.

To obtain the control law, the constant plus proportional reaching law method is applied. ${ }^{25}$ The dynamics of the switching function are directly specified by this approach which are described by the reaching function of the form

$$
\dot{S}=-Q \operatorname{sgn}(S)-K S
$$

where, $Q$ and $K$ are any positive values. A small element of $Q$ can reduce chattering, and a large $K$ chooses for a faster reaching time.

To obtain the control law, the derivative of the Eq. (9) gives

$$
\dot{S}=\lambda^{2} \dot{e}_{1}+2 \lambda \dot{e}_{2}+\dot{x}_{3}-\dot{x}_{3 d}
$$

Substituting $\dot{x}_{3}$ from Eq. (5) into Eq. (11) and gives

$$
\dot{S}=w+\hat{f}+g u+D
$$

where, $w=\lambda^{2} \dot{e}_{1}+2 \lambda \dot{e}_{2}-\dot{x}_{3 d}$

The control law, $u$ is obtained by using the reaching law method in Eqs. (10) and (12) by the necessary condition for sliding mode $\dot{S}=0$, in which the disturbance function is excluded from the equation as expressed below

$$
\begin{gathered}
-Q \operatorname{sgn}(S)-K S=w+\hat{f}+\hat{g} u \\
u=\hat{g}^{-1}(-w-\hat{f}-Q \operatorname{sgn}(S)-K S)
\end{gathered}
$$

Hence, the control law in Eq. (13) be expressed as

$$
\begin{gathered}
u=u_{e q}+u_{n} \\
u_{e q}=\hat{g}^{-1}(-w-\hat{f}-K S), u_{n}=\hat{g}^{-1}(-Q \operatorname{sgn}(S))
\end{gathered}
$$

where, $u_{e q}$ is the equivalent control for the nominal of the system in Eq. (3), and $u_{n}$ is the switching control that designed to ensure the robustness.

Since the controller is designed to achieve a better tracking performance, a smaller boundary layer is required. Hence an optimal balance between the position error and the level of control chattering can be accomplished by adjusting the thickness of the boundary layer to be $\phi>0$.

$$
u=u_{e q}+\hat{g}^{-1}(-Q s a t(S / \phi))
$$

Switching-gain, $Q$ in the control law as in Eq. (16) is designed to force all the trajectories states towards the sliding surface, $S$ and to maintain them on the surface. A constant value for the gain will guarantee the state reaches the sliding mode in spite of disturbance only in limited operating area. The controller will be more robust against the disturbance with fast reaching time and small chattering, if the switching gain, $Q$ is adaptable to the changed of the disturbance. For this purpose, the disturbance can be approximated using a disturbance observer. 
Regarding to design an adaptive switching-gain, the following reaching condition must be met to guarantee the stability of the controller.

$$
V=S(e, t) \dot{S}(e, t)<0
$$

The sliding surface condition in Eq. (9) is guaranteed when $Q>D$. Thus, the gain $Q$ can be determined by Eq. (17) and we have

$$
Q \geq \beta(w+\hat{f}+\eta+D)-w-\hat{f}
$$

where, $\eta$ is a positive constant and gain margin $\beta$ is introduced as

$$
\beta=\sqrt{g_{\max } / g_{\min }} \geq 1 \text { and } \hat{g} g^{-1} \leq \beta
$$

The switching gain of the SMC must be greater than the upper bound of the lumped disturbance. Since $D$ in Eq. (18) is assuming as a constant, approximate value for $D$ needs to be estimated.

\subsection{Disturbance observer and integration into SMC}

A nonlinear disturbance is initially designed to obtain a preliminary estimate of the unknown disturbance $D$. On the basis of the assumption that the disturbance $D$ is a slow variation system and an unknown bounded constant, it imposes

$$
\dot{D}=0
$$

Then, Eq. (5) can be rewritten

$$
D=\dot{x}_{3}(t)-\hat{f}(x)-g u(t)
$$

To estimate the unknown bounded disturbance observer, an initial disturbance observer is formulated as

$$
\begin{aligned}
\hat{D} & =-L \hat{D}+L D \\
& =-L \hat{D}+L\left(\dot{x}_{3}(t)-\hat{f}(x)-g u(t)\right)
\end{aligned}
$$

where, $L>0$ is the nonlinear gain function of the observer. The gain is constant and set by try and error. An algorithm also can be developed to determine the gain for more accurate value for $L$.

However, unavailability of $\dot{x}$ causes the disturbance observer in Eq. (22) cannot be implemented. In many systems, it may not be available due to associated measurement noise. For implementation, the initial disturbance observer above can be modified as a nonlinear disturbance observer (NDO). An auxiliary variable $z(t)$ is introduced to solve this problem.

$$
z(t)=\hat{D}-L x_{3}(t)
$$

Substituting Eqs. (22) and (23) into derivative of Eq. (23), and we have

$$
\begin{aligned}
\dot{z}(t) & =\hat{D}-L \dot{x}_{3}(t)=-L \hat{D}+L D-L \dot{x}_{3}(t) \\
& =-L z(t)-L\left(\hat{f}(x)+g u(t)+L x_{3}(t)\right)
\end{aligned}
$$

Thus, the NDO is formulated as

$$
\left\{\begin{array}{c}
\hat{D}=z(t)+L x_{3}(t) \\
\left.\dot{z}(t)=-L z(t)-L \hat{f(x)}+g(x) u(t)+L x_{3}(t)\right)
\end{array}\right.
$$

Since it is assumed that the disturbance varies slowly, it can be observed by passing it through a filter $G_{f}(s)$ of sufficiently large bandwidth in the form

$$
G_{f}(s)=\frac{1}{1+s \tau}, \tau>0
$$

Error of the NDO can be defined as

$$
e_{D}=D-\hat{D}
$$

From Eqs. (20), (22), (25) and (27), it can be shown that

$$
\begin{aligned}
\dot{e}_{D} & =\dot{D}-\hat{D} \\
& =-L(D-\hat{D})+\dot{D}=-L e_{D}+\dot{D}
\end{aligned}
$$

For $\dot{D}=0$, the estimate error Eq. (28) can be given as

$$
\dot{e}_{D}+L e_{D}=0
$$

Globally, the error $e_{D}$ is exponentially converging to zero for all $x \in$ $R^{n}$, when $L>0$ is chosen. Therefore, $D$ can be followed by the disturbance estimate $\hat{D}$. However, it may not converge to zero, when $\dot{D} \neq 0$.

By the existence of the disturbance estimate $\hat{D}$ to replace $D$, the nonlinear system equation in Eq. (5) can be rewritten as

$$
\dot{x}_{3}(t)=\hat{f}(x)+g(x) u(t)+\hat{D}
$$

Substituting $\dot{x}_{3}$ in Eq. (30) into Eq. (12) and gives

$$
\dot{S}=w+\hat{f}+g u+\hat{D}
$$

The existence of disturbance estimate function in Eq. (31) does not change the control law structure and remain as in Eq. (13). However, the control law has a different switching gain structure. The switching gain is required only to be greater than the bounds of the estimated disturbance and varied following the change of the external disturbance. This structure tends the control law to produce lower chattering in control action. Thus, the sliding surface condition will satisfy enough when the switching gain in Eq. (18) is given by

$$
Q>|\hat{D}|
$$

In the case of Eqs. (18) and (32), when $D$ is replaced by $\hat{D}$, the modified switching-gain further alleviates the chattering problem and results smoother control action.

Let the Lyapunov function candidate be given by

$$
V=V_{a}+V_{b}=0.5 S(e, t)^{2}+0.5 e_{D}^{2}
$$

The derivative of the Lyapunov function is

$$
\begin{gathered}
\dot{V}_{a}=S(e, t) \dot{S}(e, t) \leq \eta|S(e, t)| \\
\dot{V}_{b}=e_{D} \dot{e}_{D} \leq 0
\end{gathered}
$$

Substituting Eqs. (16) and (31) into Eqs. (34), and (28) into Eq. (35) yields

$$
\begin{gathered}
\dot{V}_{a}=S\left(w+\hat{f}+g \hat{g}^{-1}(-w-\hat{f}-Q \operatorname{sat}(S / \phi))+\hat{D}\right) \leq-\eta|S| \\
\dot{V}_{b}=e_{D}\left(-L e_{D}+\dot{D}\right) \leq 0
\end{gathered}
$$

Now, from Eq. (36), the discontinuous switching gain $Q$ in Eq. (18) can be rewritten as adaptation law for NDO integration. 


$$
Q \geq \beta(w+\hat{f}+\eta+\hat{D})-w-\hat{f}
$$

Existence of the disturbance observer in calculating the switching gain ensures the controller more robust and suitable for wide range of time-varying disturbance.

\subsection{Varying boundary layers}

Chattering can be decreased by introducing boundary layer, but tracking performance and robustness are compromised. Higher amplitude of chattering will guarantee more robustness controller. Consequently, it will reduce the tracking performance. Since the change of external disturbance influences the bounds of chattering, subsequently, the width of the boundary layer should also be changed to obtain an optimum tracking accuracy and smooth control input. ${ }^{26}$ Therefore, a typical formula with varying width of the boundary layers is proposed as

$$
\phi=\left\{\begin{array}{c}
\phi_{1}, e_{D} \leq \varepsilon_{1} \\
\phi_{2}, \varepsilon_{1}<e_{D}<\varepsilon_{2} \\
\phi_{3}, e_{D} \geq \varepsilon_{2}
\end{array}\right.
$$

where, $\phi_{1}, \phi_{2}$ and $\phi_{3}$ are different level of boundary layers, with $0<\phi_{1}$ $<\phi_{2}<\phi_{3} . \varepsilon_{1}$ and $\varepsilon_{2}$ are positive constant which are used to declare the switching threshold of the disturbance estimate error where $\varepsilon_{1}<\varepsilon_{2}$. The initial state will be attached to the boundary layer of sliding surface, if the initial state is outside the boundary layer, $|\mathrm{S}| \leq \phi_{3}$. The boundary layer levels changed following the formula in Eq. (39).

\section{Simulation and Experimental Setup}

In this study, the EHA system parameters are taken from the manufacturer datasheet of an existing EHA system. It is composed of a single-rod and double acting hydraulic cylinder and driven by a direct servo valve Bosch Rexroth 4WREE6, $40 \mathrm{lpm}$ flow rate at 70 bar. The dimensions of the hydraulic cylinder are $60 / 30 / 300 \mathrm{~mm}$. Original position of the piston is set in the middle of the cylinder. The EHA system parameters are listed in Table 1 and use to finely-tune the proposed control in simulation. EHA system test-bed is prepared to verify the proposed control experimentally.

Capability of the proposed control scheme is investigated by using two scenarios of test. The first test is an inspection on the effectiveness of adaptation law of integration between NDO and SMC in term of tracking precision, robustness, and smoothness of control actions. The SMC-NDO scheme employs fixed boundary layer in the control law. In this test, the SMC that applies Eq. (18) is identified as the conventional scheme, while the proposed scheme is named for the SMC that uses Eq. (36) to be integrated with NDO. The second test is an investigation on effectiveness of the varying boundary layers technique to enhance the SMC-NDO design in large change of external disturbance. In the test, the varying boundary layers technique is compared to the fixed boundary layers.

The control parameters of the SMC are $\lambda=2300, K=650, \eta=$ $1500, g_{\min }=0.75 \mathrm{xg}, g_{\max }=1.25 \mathrm{xg}, \phi_{1}=0.50, \phi_{2}=0.75, \phi_{3}=1.0, \varepsilon_{1}=$ 0.5 and $\varepsilon_{2}=0.15$. In evaluating the performance and effectiveness of the proposed control scheme, a point to point reference signal is

\begin{tabular}{|c|c|c|}
\hline \multicolumn{3}{|c|}{ Cylinder Parameters } \\
\hline$p_{s}$ & Supply pressure $(\mathrm{Pa})$ & $7.5 \times 10^{6}$ \\
\hline$V_{i 1}$ & Initial volume in chamber $1\left(\mathrm{~m}^{3}\right)$ & $0.602 \times 10^{-3}$ \\
\hline$V_{i 2}$ & Initial volume in chamber $2\left(\mathrm{~m}^{3}\right)$ & $0.496 \times 10^{-3}$ \\
\hline$A_{1}$ & Actuator ram area in chamber $1\left(\mathrm{~m}^{2}\right)$ & $2.29 \times 10^{-3}$ \\
\hline$A_{2}$ & Actuator ram area in chamber $2\left(\mathrm{~m}^{2}\right)$ & $1.58 \times 10^{-3}$ \\
\hline$x_{L}$ & Total stroke of piston $(\mathrm{m})$ & 0.3 \\
\hline$m$ & Total mass of piston and load (kg) & 20 \\
\hline$\beta_{e}$ & Effective bulk modulus $(\mathrm{Pa})$ & $1 \times 10^{9}$ \\
\hline$\rho$ & Fluid mass density $\left(\mathrm{kg} / \mathrm{m}^{2}\right)$ & 850 \\
\hline \multicolumn{3}{|c|}{ Servo Valve Parameters } \\
\hline$C_{d}$ & Discharge coefficient & 0.6 \\
\hline$w_{1}$ & Spool valve area gradient $1\left(\mathrm{~m}^{2}\right)$ & 0.02 \\
\hline$w_{2}$ & Spool valve area gradient $2\left(\mathrm{~m}^{2}\right)$ & 0.02 \\
\hline$k_{a}$ & Servo valve spool position gain $(\mathrm{m} / \mathrm{V})$ & $2.54 \times 10^{-4}$ \\
\hline \multicolumn{3}{|c|}{ Friction Parameters } \\
\hline$F_{s}$ & Stiction force $(\mathrm{N})$ & 420 \\
\hline$F_{c}$ & Coulomb friction $(\mathrm{N})$ & 310 \\
\hline$\sigma_{0}$ & Bristles stiffness coefficient $(\mathrm{N} / \mathrm{m})$ & $14 \times 10^{5}$ \\
\hline$\sigma_{1}$ & Bristles damping coefficient $\left(\mathrm{N} / \mathrm{ms}^{-1}\right)$ & 490 \\
\hline$k_{v}$ & Viscous friction $\left(\mathrm{N} / \mathrm{ms}^{-1}\right)$ & 110 \\
\hline$v_{s}$ & Stribeck velocity $(\mathrm{m} / \mathrm{s})$ & 0.02 \\
\hline \multicolumn{3}{|c|}{ Disturbance and Observer Parameters } \\
\hline$k d f$ & Load disturbance spring constant & 33,300 \\
\hline$L$ & Disturbance observer gain function & 10 \\
\hline$\tau$ & Filter time constant & 0.05 \\
\hline
\end{tabular}

Table 1 EHA system parameters

applied. This is effective to investigate the regulating ability of the controller in point to point tracking. External disturbance of this proposed work is represented by an external load disturbance. The external load disturbance proportionally changed to the piston position. Tracking performance and control activity are evaluated using sum of squared tracking error (SSTE) and sum of squared control input (SSCI), respectively. ${ }^{16}$ The SSTE is used to measure the precision of the tracking while SSCI is employed to observe the control activity in the variations of uncertainties and external disturbance. Both performance indexes are given as

$$
\begin{gathered}
S S T E=\sum_{t=0}^{N}\left(x_{1 d}(t)-\left(x_{1}(t)\right)\right)^{2} \\
S S C I=\sum_{t=0}^{N} u(t)^{2}
\end{gathered}
$$

\section{Results and Discussion}

Both the conventional SMC and the proposed SMC with adaptation laws in the integration of NDO into SMC are compared and carried out in Figs. 1 and 2. These graphical views are supported by SSTE and SSCI of both SMC as shown in Table 2.

Figs. 1 and 2 show that integration of NDO into SMC through an adaptation law tends the SMC to produce smaller switching-gain compared to the conventional one. The smaller switching-gain can be reached, since the SMC uses the NDO estimate value rather than the 

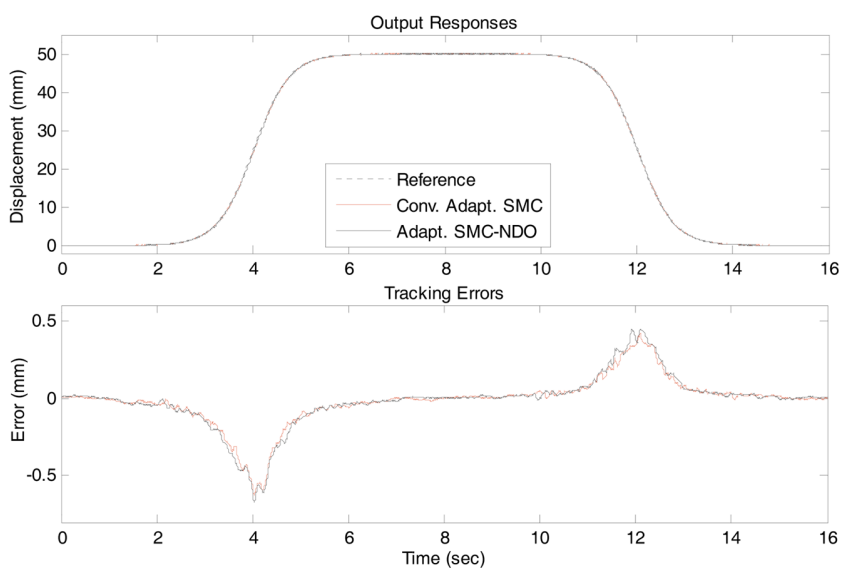

Fig. 1 Output responses and tracking errors of the SMC-NDO

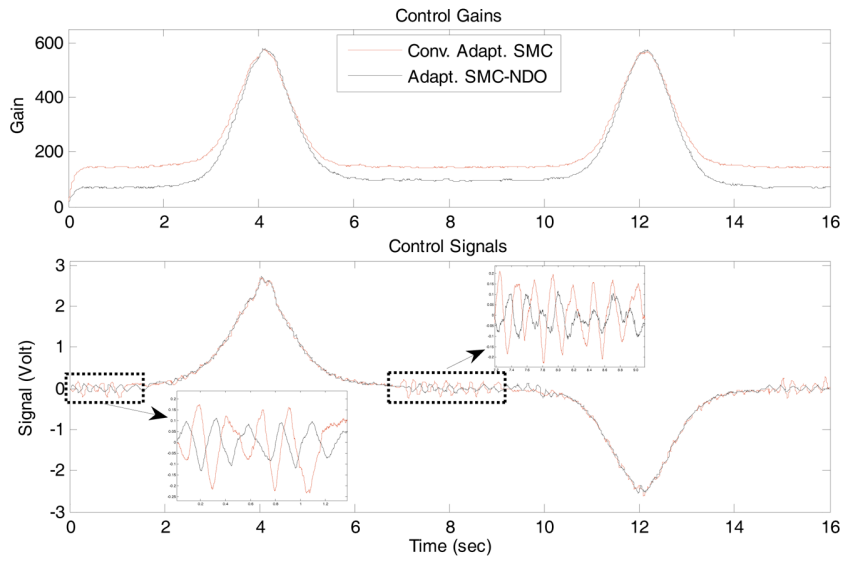

Fig. 2 Control gains and control signals of the SMC-NDO

constant value of disturbance for switching gain calculation. As a result, it minimizes chattering and chattering effect on control activities becomes linear to the change of disturbance. In another side, it may causes tracking precision of the system degrade little bit, but it is still acceptable in tolerated range. SSCI and SSTE in the first part of Table 2 indicate that the proposed SMC is capable to improve robustness and smoothness of control activity, and maintaining its tracking accuracy in a very precise level, respectively. Note that, in this step of verification both adaptive SMC employs a fixed boundary layer.

Second part of the test is an examination on effectiveness of the enhancement of the above scheme with the proposed varying boundary layers technique in a large change of the external disturbance. The test is done by generating a different reference trajectory that indicates variation of the disturbance level, and then the levels are changing significantly. Note that, the disturbance is design be linear to the reference trajectory. The trajectory and the disturbance are shown in Fig. 3.

In adaptive SMC-NDO, the change of the disturbance level as in Fig. 3 linearly influences switching gain of the SMC. Consequently, the SMC will produce different level of amplitude of chattering in the control signal following level of the disturbance. As can be seen in Fig. 4 the higher level disturbance results higher chattering than the lower
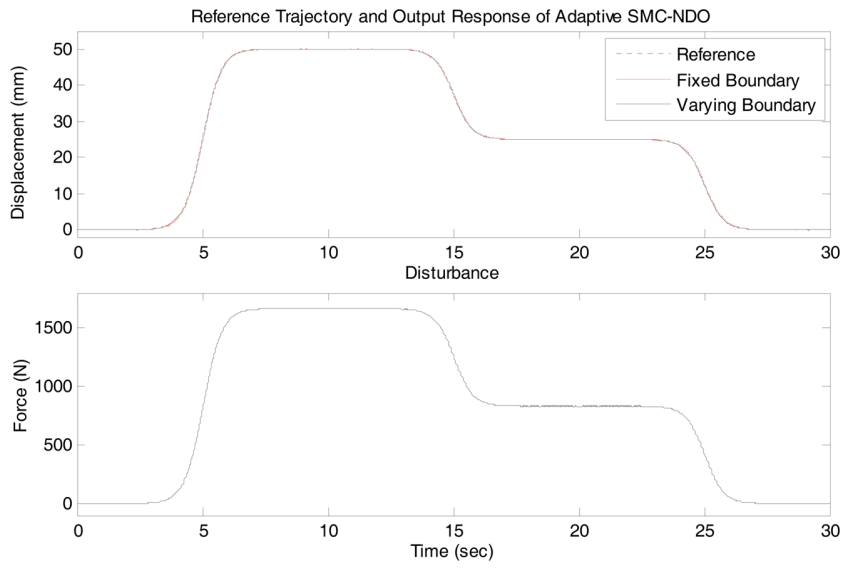

Fig. 3 Output responses and disturbance in adaptive SMC-NDO

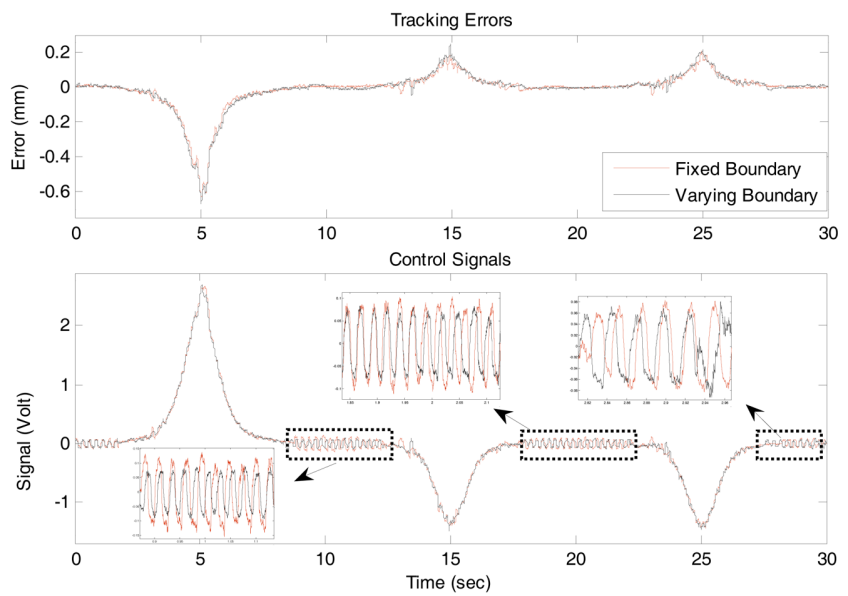

Fig. 4 The proposed scheme with varying boundary layers in a large change of external disturbance

Table 2 Comparison of the conventional and the proposed methods

\begin{tabular}{ccc}
\hline Controller Scheme & SSTE & SSCI \\
\hline Adaptive SMC & & \\
\hline Conventional & $0.602 \mathrm{e} 3$ & $1.625 \mathrm{e} 4$ \\
\hline Integrated with NDO & $0.618 \mathrm{e} 3$ & $1.608 \mathrm{e} 4$ \\
\hline Adaptive SMC-NDO with Boundary Layers & & \\
\hline Fixed Boundary & $0.649 \mathrm{e} 3$ & $1.764 \mathrm{e} 4$ \\
\hline Varying Boundary & $0.657 \mathrm{e} 3$ & $1.748 \mathrm{e} 4$ \\
\hline
\end{tabular}

level. This problem can be solved effectively by using the proposed varying boundary layer technique as shown in Fig. 4. The technique is capable to maintain amplitude of the chattering as in the whole area of the control signal around $+/-0.08 \mathrm{~V}$ in every level of disturbance. This enhancement assists the control scheme returning its ability to produce robust, precise and smooth control action. To support this visual observation, second part of Table 2 presents that the enhancement be able to reduce chattering (lower SSCI) with only a small correction in tracking accuracy (small increase of SSTE).

Based on the above preceding discussions, collaboration between SMC and NDO with new switching-gain adaptation, accomplished 
with varying boundary layers is capable and effective to result a robust and smooth control action without an excessive activity in the changes of external disturbance, and also guarantee the tracking precision of the EHA system.

\section{Conclusions}

A new scheme that represents integration of NDO into SMC with new switching-gain adaptation, and accomplished by varying boundary layers technique has been successfully proposed and verified experimentally to an EHA system that containing uncertainties and a large external disturbance. Based on the above visual views and supported by performance indexes computations, the proposed scheme ensures the control action maintains robust and smooth in the large changes of external disturbance with guaranteed tracking precision. Stability of the control scheme is guaranteed using the Lyapunov function candidate. Moreover, due to the ability to result a smooth control action, the proposed controller offers an efficiency of energy in the control of EHA systems.

\section{ACKNOWLEDGEMENT}

This research is supported by Ministry of Education (MOE) Malaysia and Universiti Teknologi Malaysia (UTM) through Research University Grant (GUP) Tier 1 vote number 05H57. Authors are grateful to the Ministry and UTM for supporting the present work.

\section{REFERENCES}

1. Liu, R. and Alleyne, A., "Nonlinear Force/Pressure Tracking of an Electrohydraulic Actuator," Journal of Dynamic Systems, Measurement, and Control - Transactions of the ASME, Vol. 122, pp. 232-237, 2000.

2. Mihajlov, M., Nikolic, V., and Antic, D., "Position Control of Electro Hydraulic Servo System using Sliding Mode Control Enhanced by Fuzzy PI Controller," Facta Universitatis Series: Mechanical Engineering, Vol. 1, No. 9, pp. 1217-1230, 2002.

3. Zeng, H. and Sepehri, N., "Tracking Control of Hydraulic Actuators Using LuGre Friction Model Compensation," Journal of Dynamic Systems, Measurement, and Control - Transactions of the ASME, Vol. 130, No. 1, pp. 1-7, 2008.

4. Loukianov, A. G., Rivera, J., Orlov, Y. V., and Teraoka E. Y. M., "Robust Trajectory Tracking for an Electrohydraulic Actuator," IEEE Transactions on Industrial Electronics, Vol. 56, No. 9, pp. 3523-3531, 2009.

5. Angue-Mintsa, H., Venugopal, R., Kenné, J. P., and Belleau C., "Adaptive Position Control of an Electrohydraulic Servo System with Load Disturbance Rejection and Friction Compensation," Journal of Dynamic Systems, Measurement, and Control Transactions of the ASME, Vol. 133, No. 6, pp. 1-8, 2011.
6. Kalyoncu, M. and Haydim, M., "Mathematical Modelling and Fuzzy Logic based Position Control of an Electrohydraulic Servo System with Internal Leakage," Mechatronics, Vol. 19, pp. 847-858, 2009.

7. Yao, B., Bu, F., Reedy, J., and Chiu, G. T. C., "Adaptive Robust Motion Control of Single-Rod Hydraulic Actuators: Theory and Experiments," IEEE/ASME Transactions on Mechatronics, Vol. 5, No. 1, pp. 79-91, 2000.

8. Khallil, H. K., "Nonlinear Systems," Prentice Hall Inc., $3^{\text {rd }}$ Ed., pp. 313-384, 2002.

9. Utkin, V., "Sliding Modes and their Applications in Variable Structure Systems," MIR Publishers, pp. 1-257, 1978.

10. Gao, W. and Hung, J. C., "A Variable Structure Control of Nonlinear Systems: A New Approach," IEEE Transactions on Industrial Electronics, Vol. 40, No. 1, pp. 45-55, 1993.

11. Bonchis, A., Corke, P. I., Rye, D. C., and Ha, Q. P., "Variable Structure Methods in Hydraulic Servos Systems Control," Automatica, Vol. 37, No. 4, pp. 589-595, 2001.

12. Liu, Y. and Handroos, H., "Technical Note Sliding Mode Control for a Class of Hydraulic Position Servo," Mechatronics, Vol. 9, No. 1, pp. 111-123, 1999.

13. Guan, C. and Pan, S., "Adaptive Sliding Mode Control of Electrohydraulic with Nonlinear Unknown Parameters," Control Engineering Practice, Vol. 16, No. 11, pp. 1275-1284, 2008.

14. Yoo, D. S. and Chung, M. J., "A Variable Structure Control with Simple Adaptation Laws for Upper Bounds on Norm of The Uncertainties," IEEE Transactions on Automatic Control, Vol. 37, No. 6, pp. 860-864, 1992.

15. Wheeler, G., Su, C. Y., and Stepanenko Y., "A Sliding Mode Control with Improved Adaptation Laws for the Upper Bounds on Norm of the Uncertainties," Automatica, Vol. 34, No. 12, pp. 1657-1661, 1998.

16. Buckner, G. D., "Intelligent Bounds on Modelling Uncertainty: Applications to Sliding Mode Control," IEEE Transactions on Systems, Man, and Cybernetics, Part C: Applications and Reviews, Vol. 32, No. 2, pp. 113-124, 2002.

17. Talole, S. E. and Phadke S. B., "Model Following Sliding Mode Control based on Uncertainty and Disturbance Estimator," Journal of Dynamic Systems, Measurement, and Control - Transactions of the ASME, Vol. 130, No. 3 pp. 1-5, 2008.

18. Chen, M. and Chen, W. H., "Sliding Mode Control for a Class of Uncertain Nonlinear System based on Disturbance Observer," International Journal of Adaptive Control and Signal Processing, Vol. 24, No. 1, pp. 51-64, 2010.

19. Rahmat, M., Zulfatman, A., Husain, K. I., Sam, Y., Ghazali, R., et al., "Modeling and Controller Design of an Industrial Hydraulic Actuator System in the Presence of Friction and Internal Leakage," International Journal of Physical Sciences, Vol. 6, No. 4, pp. 3502- 
3517, 2011.

20. Merritt, H. E., "Hydraulic Control Systems," John Wiley and Sons New York, pp. 6-118, 1967.

21. Has, Z., Rahmat, M., Husain, A., and Ghazali, R., "Sliding Mode Control with Switching-Gain Adaptation based-Disturbance Observer Applied to an Electro-Hydraulic Actuator System," Proc. of IEEE Conference on Industrial Electronics and Applications, pp. 668-673, 2013.

22. Has, Z., Rahmat, M. F. a., Husain, A. R., Ishaque, K., Ghazali, R., et al., "Robust Position Tracking Control of an Electro-hydraulic Actuator in the Presence of Friction and Internal Leakage," Arabian Journal for Science and Engineering, Vol. 39, No. 4, pp. 2965-2978, 2014.

23. Canudas de Wit, C., Olsson, H., Astrom, K. J., and Lischinsky, P., "A New Model for Control of Systems with Friction," IEEE Transactions on Automatic Control, Vol. 40, No. 3, pp. 419-425, 1995.

24. Olsson, H., Åström, K. J., Canudas de Wit, C., Gäfvert, M., and Lischinsky, P., "Friction Models and Friction Compensation," European Journal of Control, Vol. 4, No. 3, pp. 176-195, 1998.

25. Hung, J. Y., Gao, W., and Hung, J. C., "Variable Structure Control: A Survey," IEEE Transactions on Industrial Electronics, Vol. 40, No. 1, pp. 2-22, 1993.

26. Chen, H.-M., Renn, J.-C., and Su, J.-P., "Sliding Mode Control with Varying Boundary Layers for an Electro-Hydraulic Position Servo System," International Journal of Advanced Manufacturing Technology, Vol. 26, No. 1-2, pp. 117-123, 2005.

\section{APPENDIX}

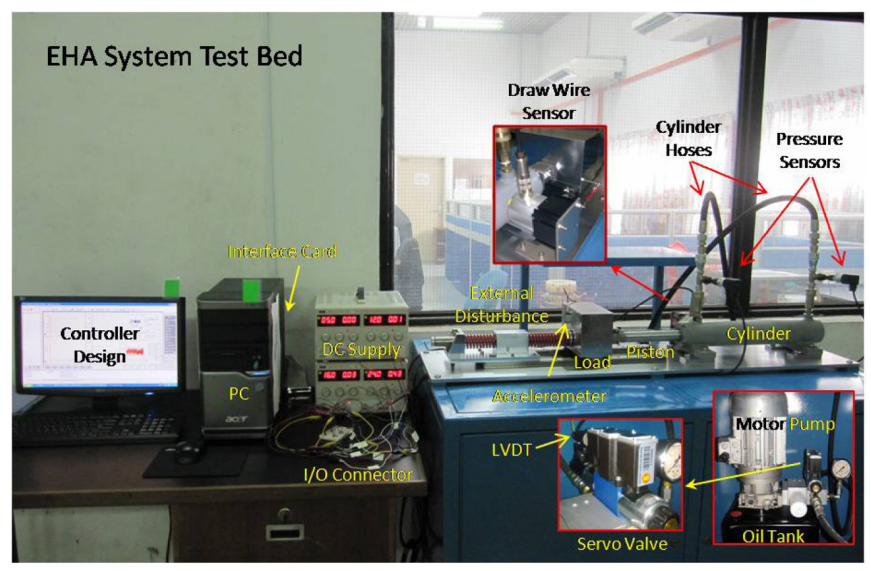

